\title{
Aspects of a supersymmetric Brans-Dicke theory
}

\author{
Riccardo Catena \\ Deutsches Elektronen-Syncrotron (DESY), \\ 22603 Hamburg, Germany \\ (catena@mail.desy.de)
}

\begin{abstract}
We consider a locally supersymmetric theory where the Planck mass is replaced by a dynamical superfield. This model can be thought of as the Minimal Supersymmetric extension of the Brans-Dicke theory (MSBD). The motivation that underlies this analysis is the research of possible connections between Dark Energy models based on Brans-Dicke-like theories and supersymmetric Dark Matter scenarios. We find that the phenomenology associated with the MSBD model is very different compared to the one of the original Brans-Dicke theory: the gravitational sector does not couple to the matter sector in a universal metric way. This feature could make the minimal supersymmetric extension of the BD idea phenomenologically inconsistent.
\end{abstract}

\section{INTRODUCTION}

During the last decade of cosmological observations, the picture of a Universe dominated by Dark Matter and Dark Energy has emerged [1]. Although a real understanding of the microscopic nature of such cosmological components is still missing, different theories are at present under analysis.

Concerning Dark Matter particles, their indirectly observed interaction properties naturally fit those of the lightest state of supersymmetric models with conserved $R$-parity [2]. If supersymmetry really exists, a simple and well motivated cosmological model should include a supersymmetric dark matter particle.

Dark Energy interaction properties are even more obscure than the Dark Matter ones. An example of this lack of knowledge is the difficulty to explain the extremely small value of its mass scale that, in a phenomenologically consistent model, should be of the order of the present value of the Hubble parameter [3]. As a consequence, direct couplings of Dark Energy to matter fields are strongly constrained by fifth force searches [4]. A possible way to avoid such constraints is to work in the framework of Scalar-Tensor theories [5]. In these theories the gravitational interaction is described in terms of both a metric tensor and a scalar field. Moreover, the energy density of this extra scalar degree of freedom can be easily identified with Dark Energy [6]. An interesting feature of such models is that the gravitational sector (that also includes the Dark Energy scalar) couples to the matter sector in a universal metric way so that fifth force bounds are satisfied by construction [4]. If Dark Energy has a scalar nature, Scalar-Tensor theories provide a natural framework to discuss its properties.

The interesting possibility to relate a Scalar-Tensor interpretation of Dark Energy to a supersymmetric description of Dark Matter leads to study supersymmetric extensions of Scalar-Tensor theories. This is the topic of the present paper. As we will see, the results of this analysis do not rely on the particular choice of the underlying Scalar-Tensor theory; for this reason we will consider the simplest one, i.e. the Brans-Dicke (BD) theory [7].
In the BD theory the Planck mass is replaced by a dynamical scalar field. In this paper we consider the supersymmetric analogous of this mechanism: we replace in the supergravity Lagrangian the Planck mass with a chiral superfield, the "Planck superfield". Such a replacement defines the "natural" supersymmetric extension of the BD theory. Let us refer to it as the Minimal Supersymmetric Brans-Dicke theory (MSBD) to distinguish it from other possible approaches. We find that, contrary to the original BD theory, in the MSBD the gravitational sector does not couple to the matter sector in a universal metric way. As a result, possible violations of the weak equivalence principle could make the minimal supersymmetric extension of the BD idea phenomenologically inconsistent.

In spite of this conclusion, we find the subject a good laboratory for studying realistic models of Dark MatterDark Energy unification. For instance, alternative approaches to the problem could provide a consistent scenario where Dark Matter and Dark Energy are identified with different components of the Planck multiplet.

The plan of this paper is as follows. In section II and III we introduce notation and review the BD model and the concept of universal metric coupling. Section IV is devoted to the MSBD theory; we will specially underline the differences between its phenomenology and the one of the original BD theory. Section $\mathrm{V}$ is concerned with some technical details, related with the component fields formalism, that should make the arguments of section IV more precise. The results are discussed in section VI. Finally, we list in the appendix useful expressions that we used during the computations.

\section{NOTATION}

We will use in the following the same notation and conventions of [8]. We list here for clarity some of them.

The superspace is described in terms of the coordinates $\left(y^{m}, \theta_{\alpha}\right)$. Greek indexes label two components Weyl spinors while latin indexes the components of fourvectors. Indexes transforming under local coordinates 
transformations in superspace are called Einstein indexes and are taken from the end of the alphabet, for example $(m, n, \ldots)$. Instead, indexes transforming under local Lorentz transformations are called Lorentz indexes and are taken from the beginning of the alphabet, for example $(a, b, \ldots)$. The power series expansion in $\theta_{\alpha}$ of a chiral superfield $\Phi$ is given by

$$
\Phi\left(y^{m}, \theta_{\alpha}\right)=A\left(y^{m}\right)+\sqrt{2} \theta^{\alpha} \chi_{\alpha}\left(y^{m}\right)+\theta^{\alpha} \theta_{\alpha} F\left(y^{m}\right)
$$

where $A\left(y^{m}\right)$ and $F\left(y^{m}\right)$ are complex scalars and $\chi_{\alpha}\left(y^{m}\right)$ a Weyl spinor. We will couple matter superfields to the minimal supergravity multiplet. This contains the vielbein $e_{m}^{a}$, the gravitino $\psi_{\alpha}^{a}$ and two auxiliary fields: a vector $b^{a}$ and a scalar $M$. Finally, covariant derivatives with respect to supergravity transformations are denoted by $\mathcal{D}_{\alpha}, \overline{\mathcal{D}}^{\dot{\alpha}}$ and $\mathcal{D}_{m}$.

\section{THE BRANS-DICKE THEORY AND THE UNIVERSAL METRIC COUPLING}

In General Relativity the coupling between gravity and matter is described by the following Lagrangian

$$
\mathcal{L}_{\mathrm{EH}}=-\frac{1}{2} e M_{\mathrm{Pl}}^{2} \mathcal{R}+\mathcal{L}_{\mathrm{M}}\left[e_{m}^{a}, \Psi\right]
$$

where $e \equiv \operatorname{det}\left(e_{m}^{a}\right), \mathcal{R}$ is the Ricci scalar and $\Psi$ symbolically represents all matter fields involved in the theory. In the BD approach to the gravitational interaction the Planck mass appearing in eq. (2) becomes dynamical by means of the substitution

$$
M_{\mathrm{Pl}}^{2} \Longrightarrow \varphi^{2}\left(y^{m}\right)
$$

where $\varphi\left(y^{m}\right)$ is a real scalar field. As a consequence eq. (2) is replaced by

$$
\begin{aligned}
\mathcal{L}_{\mathrm{BD}} & =\mathcal{L}_{\varphi}\left[e_{m}^{a}, \varphi\right]+\mathcal{L}_{\mathrm{M}}\left[e_{m}^{a}, \Psi\right] \\
& =-\frac{1}{2} e\left(\varphi^{2} \mathcal{R}+\omega \partial_{m} \varphi \partial^{m} \varphi\right)+\mathcal{L}_{\mathrm{M}}\left[e_{m}^{a}, \Psi\right],
\end{aligned}
$$

where the factor $\omega$ that multiplies the kinetic term of $\varphi$ has to be tuned to fit the post-newtonian bounds [9]. Eq. (4) gives the so called "Jordan frame" formulation of the theory. In this frame the BD scalar does not appear in the matter Lagrangian and particle physics is just the standard one. The theory can be formulated in other frames related to the Jordan one by a Weyl rescaling of the vielbein such as $e_{m}^{a} \rightarrow e_{m}^{a} e^{l(\varphi)}$, where $l(\varphi)$ is some $\varphi$-dependent function. In these alternative formulations the matter Lagrangian acquires an explicit functional dependence from $\varphi$, i.e. $\mathcal{L}_{\mathrm{M}}=\mathcal{L}_{\mathrm{M}}\left[e_{m}^{a} e^{l(\varphi)}, \Psi\right]$. However, the inverse Weyl rescaling $e_{m}^{a} \rightarrow e_{m}^{a} e^{-l(\varphi)}$ always brings back the theory to its original version in which particle physics is just the standard one.

Eq. (4) shows that in the BD theory all matter fields feel the gravitational interaction through the same vielbein, the Jordan frame vielbein. For this reason such a matter-gravity coupling is also called universal and metric. This is a non trivial property and has very important phenomenological implications. It can be shown, for instance, that in a theory where matter couples to gravity in a universal metric way the weak equivalence principle is satisfied by construction [4].

A typical example of non universal metric coupling is the following. Let us introduce in the gravitational sector a long range scalar field $\phi$ that couples like a dilaton to the field strength $F_{\mu \nu}$ of some (for simplicity) abelian gauge group with gauge coupling $\bar{g}$

$$
S_{\phi \mathrm{FF}}=-\frac{1}{4 \bar{g}^{2}} \int d^{4} x \sqrt{-g} g^{\alpha \mu} g^{\beta \nu} \phi F_{\alpha \beta} F_{\mu \nu}
$$

where $g_{\mu \nu}$ is the metric tensor and $g \equiv \operatorname{det}\left(g_{\mu \nu}\right)$. Since in four dimensions the combination $\sqrt{-g} g^{\alpha \mu} g^{\beta \nu}$ is Weyl invariant, the scalar field $\phi$ can not be reabsorbed by means of a rescaling of the metric. Therefore, in this example the gauge field strength $F_{\mu \nu}$ feels gravity through the metric $g_{\mu \nu}$ and the scalar $\phi$. In other words, since no Weyl rescaling of $g_{\mu \nu}$ can "remove" $\phi$ from the matter sector $\left(S_{\phi \mathrm{FF}}\right)$, it is not possible in this case to define a Jordan frame. Such a non metric and universal coupling can be easily interpreted in terms of an effective, scalarfield dependent, gauge coupling, i.e. $\bar{g}_{\text {eff }}^{-2}(\phi) \equiv \bar{g}^{-2} \phi$. Moreover, it can be shown that in this picture also the masses of the particles become $\phi$-dependent. However the proton and neutron masses, for instance, acquire different dependences from $\phi$. This is a consequence of the fact that a gauge interaction contributes differently to the proton and neutron binding energies. As a result, the theory manifestly violates the weak equivalence principle [4].

\section{THE MINIMAL SUPERSYMMETRIC BRANS-DICKE THEORY}

Eq. (3) gives a prescription to construct the BD Lagrangian starting from the Einstein-Hilbert one. In this section we apply an analogous prescription to the supergravity Lagrangian

$$
\mathcal{L}_{\mathrm{sg}}=-3 M_{\mathrm{Pl}}^{2} \int d^{2} \theta 2 \mathcal{E} R+\mathcal{L}_{\mathrm{M}}[H, \Psi]+\text { h.c. },
$$

where $H$ is the supergravity multiplet, $\mathcal{E}$ is the chiral density and $R$ represents the curvature superfield, defined as the covariant derivative of the spin connection.

Let us start introducing a chiral superfield $\Phi$ with components given in the power series expansion (1). We will call $\Phi$ the Planck superfield. This dynamical object allows the natural supersymmetric extension of the substitution (3)

$$
M_{\mathrm{Pl}}^{2} \Longrightarrow \Phi^{2}\left(y^{m}, \theta_{\alpha}\right)
$$


Applying the substitution (7) to eq. (6) one finds

$$
\begin{aligned}
\mathcal{L}_{\mathrm{MSBD}} & =\mathcal{L}_{\Phi}[H, \Phi]+\mathcal{L}_{\mathrm{M}}[H, \Psi] \\
& =-3 \int d^{2} \theta \Phi^{2} 2 \mathcal{E} R- \\
& -\frac{1}{8} \int d^{2} \theta 2 \mathcal{E}\left(\overline{\mathcal{D}}_{\dot{\alpha}} \overline{\mathcal{D}}^{\dot{\alpha}}-8 R\right) \Phi^{\dagger} \Phi+ \\
& +\mathcal{L}_{M}[H, \Psi]+\text { h.c. },
\end{aligned}
$$

where in the third line, in analogy with eq. (4), we introduced a kinetic term for $\Phi$. To be as general as possible we do not assume any particular form for $\mathcal{L}_{M}$.

Eq. (8) defines the Minimal Supersymmetric Brans Dicke theory (MSBD). Its invariance under supergravity transformations follows from the properties of chiral densities. By definitions, chiral densities transform like total derivatives in the space $\left(y^{m}, \theta_{\alpha}\right)$ and the product of a chiral density and a chiral superfield is again a chiral density [8]. Moreover, the superfields $(\overline{\mathcal{D}} \mathcal{D}-8 R) \Phi^{\dagger} \Phi$ and $\Phi^{2}$ are chiral if $\Phi$ is chiral. This proves the invariance of the Lagrangian (8) under supergravity transformations.

Let us focus now on its phenomenology. As we will see explicitly in the next section, the component fields expansion of eq. (8) gives rise to a Lagrangian with the following structure

$$
\begin{aligned}
\mathcal{L}_{\mathrm{MSBD}} & =\mathcal{L}_{\Phi}\left[e_{m}^{a}, \psi_{\alpha}^{a}, b^{a}, M, A, \chi_{\alpha}, F\right] \\
& +\mathcal{L}_{\mathrm{M}}\left[e_{m}^{a}, \psi_{\alpha}^{a}, b^{a}, M, \Psi\right]
\end{aligned}
$$

where each fields was already introduced during the previous sections. Eq. (9) is the supersymmetric version of eq. (4). The crucial difference between the two Lagrangians is that in the supersymmetric one $\mathcal{L}_{\mathrm{M}}$ and $\mathcal{L}_{\Phi}$ communicate also through the auxiliary fields $b^{a}$ and $M$. This has deep phenomenological consequences when the auxiliary fields are removed by means of their equations of motion. To show this point, let us write the general solution of the equations of motion for $M$ and $b^{a}$ as follows

$$
\begin{aligned}
b^{a} & =h_{1}\left(\ldots, A, \chi_{\alpha}\right), \\
M & =h_{2}\left(\ldots, A, \chi_{\alpha}\right),
\end{aligned}
$$

where $h_{1}$ and $h_{2}$ are two appropriate functions of the fields involved in the theory. In eq. (10) we underlined the crucial dependence of $h_{1}$ and $h_{2}$ from $A$ and $\chi_{\alpha}$. Now, replacing the solutions (10) in the Lagrangian (9), the degrees of freedom of the Planck multiplet explicitly appear in the matter Lagrangian. Since no Weyl rescaling of the vielbein can remove the auxiliary fields from $\mathcal{L}_{\mathrm{M}}$, it follows that the Planck multiplet couples intrinsically to matter. Therefore, there is no way to write the matter Lagrangian as $\mathcal{L}_{\mathrm{M}}\left[e_{m}^{a}, \psi_{\alpha}^{a}, \Psi\right]$ by means of a suitable vielbein redefinition of the form $e_{m}^{a} \rightarrow e_{m}^{a} e^{l\left(A, \chi_{\alpha}, F\right)}$, where $l$ is an appropriate function of the components of $\Phi$. In other words, a Jordan frame does not exist for such a theory. The main consequence is that in the MSBD theory the weak equivalence principle is not satisfied by construction and time variations of masses and couplings are not under control. In the next section we will give the explicit expressions for eqs. (9) and (10).

\section{COMPONENT FIELDS}

The Lagrangians given in this section are obtained using the results summarized in the appendix. Let us start with the first term of eq. (8). Its component fields expansion reads

$$
\begin{aligned}
& -3 \int d^{2} \theta \Phi^{2} 2 \mathcal{E} R+\text { h.c. }= \\
& -\frac{1}{4} e\left(A^{2}+A^{2 *}\right) \mathcal{R} \\
& +\frac{1}{2} e \varepsilon^{a b c d}\left(\bar{\psi}_{a} \bar{\sigma}_{b} \mathcal{D}_{c} \psi_{d} A^{2}-\psi_{a} \sigma_{b} \mathcal{D}_{c} \bar{\psi}_{d} A^{2 *}\right) \\
& +\frac{1}{16} e\left(A^{2}-A^{2 *}\right) \varepsilon^{a b c d}\left(\bar{\psi}_{a} \bar{\sigma}_{b} \mathcal{D}_{c} \psi_{d}+\psi_{a} \sigma_{b} \mathcal{D}_{c} \bar{\psi}_{d}\right) \\
& -\frac{1}{\sqrt{2}} e A \chi \sigma^{a} \bar{\sigma}^{b} \psi_{a b}-\frac{1}{\sqrt{2}} e A^{*} \bar{\chi} \bar{\sigma}^{a} \sigma^{b} \bar{\psi}_{a b} \\
& -\frac{1}{6} e\left(A^{2}+A^{2 *}\right) M M^{*}+\frac{1}{6} e\left(A^{2}+A^{2 *}\right) b^{a} b_{a} \\
& -\frac{i}{2} e e_{a}^{m} \mathcal{D}_{m} b^{a}\left(A^{2}-A^{2 *}\right) \\
& -\frac{1}{4} e \psi_{a} \sigma^{a} \bar{\psi}_{b} b^{b}\left(A^{2}-A^{2 *}\right)-\frac{1}{4} e \bar{\psi}_{a} \bar{\sigma}^{a} \psi_{b} b^{b}\left(A^{2}-A^{2 *}\right) \\
& -\frac{i}{\sqrt{2}} e A \chi \psi_{a} b^{a}+\frac{i}{\sqrt{2}} e A^{*} \bar{\chi} \bar{\psi}_{a} b^{a} \\
& -\frac{1}{2} e \chi \chi M-\frac{1}{2} e \bar{\chi} \bar{\chi} M^{*}+e A F M+e A^{*} F^{*} M^{*},(11)
\end{aligned}
$$

where

$$
\begin{aligned}
& \psi_{n m}^{\alpha}=\mathcal{D}_{n} \psi_{m}^{\alpha}-\mathcal{D}_{m} \psi_{n}^{\alpha}, \\
& \mathcal{D}_{n} \psi_{m}^{\alpha}=\partial_{n} \psi_{m}^{\alpha}+\psi_{m}^{\beta} \omega_{n \beta}^{\alpha},
\end{aligned}
$$

and $\omega_{n \beta}{ }^{\alpha}$ is the algebra-valued spin connection.

Now we focus on the kinetic term of the Planck superfield. Its component fields expansion is given by

$$
\begin{aligned}
& -\frac{1}{8} \int d^{2} \theta 2 \mathcal{E}\left(\overline{\mathcal{D}}_{\dot{\alpha}} \overline{\mathcal{D}}^{\dot{\alpha}}-8 R\right) \Phi^{\dagger} \Phi= \\
& +\frac{1}{6} e|A|^{2} \mathcal{R}-e \partial_{m} A \partial^{m} A^{*} \\
& -\frac{i}{2} e\left(\chi \sigma^{m} \mathcal{D}_{m} \bar{\chi}+\bar{\chi} \bar{\sigma}^{m} \mathcal{D}_{m} \chi\right) \\
& -\frac{1}{6} e|A|^{2} \varepsilon^{a b c d}\left(\bar{\psi}_{a} \bar{\sigma}_{b} \mathcal{D}_{c} \psi_{d}-\psi_{a} \sigma_{b} \mathcal{D}_{c} \bar{\psi}_{d}\right) \\
& +\frac{\sqrt{2}}{3} e\left(A^{*} \chi \sigma^{a b} \psi_{a b}+A \bar{\chi} \bar{\sigma}^{a b} \bar{\psi}_{a b}\right) \\
& -\frac{\sqrt{2}}{2} e\left(\bar{\psi}_{a} \bar{\sigma}^{b} \sigma^{a} \bar{\chi} \partial_{b} A+\chi \sigma^{a} \bar{\sigma}^{b} \psi_{a} \partial_{b} A^{*}\right) \\
& +\frac{1}{4} e \varepsilon^{a b c d}\left(A^{*} \partial_{a} A-A \partial_{a} A^{*}\right) \psi_{b} \sigma_{c} \bar{\psi}_{d}
\end{aligned}
$$




$$
\begin{aligned}
& -\frac{1}{9} e|A|^{2} b_{a} b^{a}+\frac{i}{3} e b^{a}\left(A \partial_{a} A^{*}-A^{*} \partial_{a} A\right) \\
& -\frac{1}{6} e \chi \sigma^{a} b_{a} \bar{\chi}-i \frac{\sqrt{2}}{6} e b^{a}\left(A \bar{\psi}_{a} \bar{\chi}-A^{*} \psi_{a} \chi\right) \\
& +e F F^{*}+\frac{1}{9} e|A|^{2}|M|^{2}-\frac{1}{3} e M A^{*} F-\frac{1}{3} e M^{*} F^{*} A \\
& +\mathcal{L}_{4},
\end{aligned}
$$

where $\mathcal{L}_{4}$ includes only 4 -fermions interactions and it will be given afterwords.

Using eqs. (11) and (12) one can write the explicit component fields expansion of eq. (8). For simplicity we decompose the final Lagrangian as follows

$$
\mathcal{L}_{\mathrm{MSBD}}=\mathcal{L}_{\mathrm{K}}+\mathcal{L}_{\text {int }}+\mathcal{L}_{4}+\mathcal{L}_{\text {aux }},
$$

where $\mathcal{L}_{\mathrm{K}}$ is the Lagrangian for the kinetic terms of the fields contained in the Planck and supergravity multiplets, $\mathcal{L}_{\text {int }}$ describes the interactions between the Planck and supergravity multiplets not included in $\mathcal{L}_{4}$ and $\mathcal{L}_{\text {aux }}$ is the Lagrangian for the auxiliary fields where we also absorbed $\mathcal{L}_{\mathrm{M}}$. We list in the following their explicit expressions. $\mathcal{L}_{\mathrm{K}}$ reads

$$
\begin{aligned}
\mathcal{L}_{\mathrm{K}} & =-\frac{1}{4} e f\left(A, A^{*}\right) \mathcal{R}-e \partial_{m} A \partial^{m} A^{*} \\
& -\frac{i}{2} e\left(\chi \sigma^{m} \mathcal{D}_{m} \bar{\chi}+\bar{\chi} \bar{\sigma}^{m} \mathcal{D}_{m} \chi\right) \\
& +e \varepsilon^{a b c d}\left[g_{1}\left(A, A^{*}\right) \bar{\psi}_{a} \bar{\sigma}_{b} \mathcal{D}_{c} \psi_{d}\right. \\
& \left.+g_{2}\left(A, A^{*}\right) \psi_{a} \sigma_{b} \mathcal{D}_{c} \bar{\psi}_{d}\right]
\end{aligned}
$$

where the functions $f, g_{1}$ and $g_{2}$ are defined as follows

$$
\begin{aligned}
f\left(A, A^{*}\right) & =A^{2}+A^{2 *}-\frac{2}{3}|A|^{2}, \\
g_{1}\left(A, A^{*}\right) & =\frac{9}{16} A^{2}-\frac{1}{16} A^{2 *}-\frac{1}{6}|A|^{2}, \\
g_{2}\left(A, A^{*}\right) & =\frac{1}{16} A^{2}-\frac{9}{16} A^{2 *}+\frac{1}{6}|A|^{2} .
\end{aligned}
$$

The Lagrangian $\mathcal{L}_{\text {int }}$ is given by

$$
\begin{aligned}
\mathcal{L}_{\text {int }} & =\frac{\sqrt{2}}{3} e\left(A^{*} \chi \sigma^{a b} \psi_{a b}+A \bar{\chi} \bar{\sigma}^{a b} \bar{\psi}_{a b}\right) \\
& -\frac{\sqrt{2}}{2} e\left(\bar{\psi}_{a} \bar{\sigma}^{b} \sigma^{a} \bar{\chi} \partial_{b} A+\chi \sigma^{a} \bar{\sigma}^{b} \psi_{a} \partial_{b} A^{*}\right) \\
& +\frac{1}{4} e \varepsilon^{a b c d}\left(A^{*} \partial_{a} A-A \partial_{a} A^{*}\right) \psi_{b} \sigma_{c} \bar{\psi}_{d} \\
& -\frac{1}{\sqrt{2}} e A \chi \sigma^{a} \bar{\sigma}^{b} \psi_{a b}-\frac{1}{\sqrt{2}} e A^{*} \bar{\chi} \bar{\sigma}^{a} \sigma^{b} \bar{\psi}_{a b} .
\end{aligned}
$$

The 4-fermions interactions $\mathcal{L}_{4}$ read

$$
\begin{aligned}
\mathcal{L}_{4} & =+\frac{1}{4} e \chi \sigma^{c} \bar{\sigma}^{b} \psi_{c} \bar{\psi}_{b} \bar{\chi}-i \frac{e \sqrt{2}}{8} \bar{\psi}_{a}\left(\bar{\sigma}^{b} \eta^{a c}\right. \\
& \left.+\bar{\sigma}^{a} \sigma^{c} \bar{\sigma}^{b}\right) \psi_{c} \bar{\psi}_{b} \bar{\chi} A+\text { h.c. },
\end{aligned}
$$

and finally,

$$
\begin{aligned}
& \mathcal{L}_{\text {aux }}=\frac{1}{6} e f\left(A, A^{*}\right) b_{a} b^{a}+\frac{i}{3} e b^{a}\left(A \partial_{a} A^{*}-A^{*} \partial_{a} A\right) \\
& -\frac{1}{6} e \chi \sigma^{a} b_{a} \bar{\chi}-i \frac{\sqrt{2}}{6} e b^{a}\left(A \bar{\psi}_{a} \bar{\chi}-A^{*} \psi_{a} \chi\right) \\
& -\frac{i}{2} e e_{a}^{m} \mathcal{D}_{m} b^{a}\left(A^{2}-A^{2 *}\right) \\
& -\frac{1}{4} e \psi_{a} \sigma^{a} \bar{\psi}_{b} b^{b}\left(A^{2}-A^{2 *}\right)-\frac{1}{4} e \bar{\psi}_{a} \bar{\sigma}^{a} \psi_{b} b^{b}\left(A^{2}-A^{2 *}\right) \\
& -\frac{i}{\sqrt{2}} e A \chi \psi_{a} b^{a}+\frac{i}{\sqrt{2}} e A^{*} \bar{\chi} \bar{\psi}_{a} b^{a} \\
& -\frac{1}{6} e f\left(A, A^{*}\right)|M|^{2}+e F F^{*}-\frac{1}{3} e M A^{*} F-\frac{1}{3} e M^{*} F^{*} A \\
& -\frac{1}{2} e \chi \chi M-\frac{1}{2} e \bar{\chi} \bar{\chi} M^{*}+e A F M+e A^{*} F^{*} M^{*} \\
& +\mathcal{L}_{\mathrm{M}}\left[e_{m}^{a}, \psi_{\alpha}^{a}, b^{a}, M, \Psi\right] .
\end{aligned}
$$

To recover a complete analogy with eq. (4) one has to perform in eq. (13) a Weyl rescaling of the vielbein in order to have a kinetic term for the graviton of the form $-1 / 2 e|A|^{2} \mathcal{R}$. However, $\mathcal{L}_{\mathrm{M}}$ also includes a contribution proportional to $\mathcal{R}$; as a consequence such a rescaling should be performed only after having specified $\mathcal{L}_{\mathrm{M}}$. Adding $\mathcal{L}_{\mathrm{M}}$ to eq. (11) and taking the limit $A=A^{*}=M_{\mathrm{Pl}}$ and $F=\chi=0$, one gets the expression

$$
\begin{aligned}
& -\frac{1}{2} e M_{\mathrm{Pl}}^{2} \mathcal{R} \\
& +\frac{1}{2} e M_{\mathrm{Pl}}^{2} \varepsilon^{a b c d}\left(\bar{\psi}_{a} \bar{\sigma}_{b} \mathcal{D}_{c} \psi_{d}-\psi_{a} \sigma_{b} \mathcal{D}_{c} \bar{\psi}_{d}\right) \\
& -\frac{1}{3} e M_{\mathrm{Pl}}^{2} M M^{*}+\frac{1}{3} e M_{\mathrm{Pl}}^{2} b^{a} b_{a} \\
& +\mathcal{L}_{\mathrm{M}}\left[e_{m}^{a}, \psi_{\alpha}^{a}, b^{a}, M, \Psi\right] .
\end{aligned}
$$

that, in agreement with [8], gives the component fields expansion of the Lagrangian (6).

As usual, auxiliary fields can be expressed in terms of other fields involved in the theory by means of their equations of motion. Using the Lagrangian (18) one finds

$$
\begin{aligned}
& b_{a}=-i \frac{1}{f}\left(A^{*} \partial_{a} A-A^{*} \partial_{a} A\right)+\frac{1}{2 f} \chi \sigma_{a} \bar{\chi} \\
& +i \frac{\sqrt{2}}{2 f}\left(A \overline{\psi_{a}} \bar{\chi}-A^{*} \psi_{a} \chi\right)+i \frac{3}{\sqrt{2} f}\left(A \chi \psi_{a}-A^{*} \bar{\chi} \bar{\psi}_{a}\right) \\
& +\frac{3}{4 f}\left(A^{2}-A^{2 *}\right)\left(\psi_{a} \sigma^{a} \bar{\psi}_{b}+\bar{\psi}_{a} \bar{\sigma}^{a} \psi_{b}\right) \\
& +i \frac{3}{2 f} \omega_{m a}^{m}-i \frac{3}{2 f} \partial_{m}\left[e_{a}^{m}\left(A^{2}-A^{2 *}\right)\right] \\
& -\frac{3}{e f} \frac{\partial \mathcal{L}_{\mathrm{M}}}{\partial b^{a}} \\
& M=C_{1}\left(-3 \bar{\chi} \bar{\chi}+\frac{6}{e} \frac{\partial \mathcal{L}_{\mathrm{M}}}{\partial M^{*}}\right) \\
& F^{*}=C_{2}\left(-3 \bar{\chi} \bar{\chi}+\frac{6}{e} \frac{\partial \mathcal{L}_{\mathrm{M}}}{\partial M^{*}}\right)
\end{aligned}
$$


where

$$
\begin{aligned}
C_{1}\left(A, A^{*}\right) & \equiv \frac{1}{6|A|^{2}-A^{2}-A^{2 *}}, \\
C_{2}\left(A, A^{*}\right) & \equiv C_{1}\left(A, A^{*}\right)\left(\frac{1}{3} A^{*}-A\right) .
\end{aligned}
$$

In eqs. (20) we omitted the dependence from $A$ and $A^{*}$ of the functions $f, C_{1}$ and $C_{2}$. When $\mathcal{L}_{\mathrm{M}}$ is specified, from eqs. (20) one can explicitly compute the functions $h_{1}$ and $h_{2}$ introduced in section IV.

\section{CONCLUSIONS}

In this paper we have studied the minimal supersymmetric extension of the BD theory (MSBD) defined by eq. (8). The underlying motivation was the research of possible connections between a Scalar-Tensor interpretation of Dark Energy and a supersymmetric description of Dark Matter. Eq. (8) is obtained replacing the Planck mass with a chiral superfield in the supergravity Lagrangian (6). We called this extra superfield the Planck superfield. Although this approach looks very natural, the resulting phenomenology is radically different from the one of the original BD theory. In the MSBD theory the extra degrees of freedom of the Planck superfield intrinsically couple to matter and a Jordan frame formulation can not be achieved through a suitable vielbein redefinition. As a consequence, this theory does not satisfy the weak equivalence principle by construction. This conclusion could make the minimal supersymmetric extension of the BD idea phenomenologically inconsistent. 1

In spite of this result, we find that if a consistent supersymmetric Scalar-Tensor theory were constructed, it could provide a natural framework to achieve a Dark Matter-Dark Energy unification. For instance, in such a scenario Dark Matter and Dark Energy could be identified with different components of the Planck superfield. This issue is at present under analysis.

\section{APPENDIX}

We list here some useful $\theta$ expansions that we used for deriving the Lagrangians of section V. Let us start with the chiral density $\mathcal{E}$. Its component fields expansion is given by [8]

$$
2 \mathcal{E}=e\left[1+i \theta \sigma^{a} \bar{\psi}_{a}-\theta \theta\left(M^{*}+\bar{\psi}_{a} \bar{\sigma}^{a b} \bar{\psi}_{b}\right)\right] .
$$

The curvature superfield has the following power series expansion [8]

$$
\begin{aligned}
R & =-\frac{1}{6}\left\{M+\theta\left(\sigma^{a} \bar{\sigma}^{b} \psi_{a b}-i \sigma^{a} \bar{\psi}_{a} M+i \psi_{a} b^{a}\right)\right. \\
& +\theta \theta\left[-\frac{1}{2} \mathcal{R}+i \bar{\psi}^{a} \bar{\sigma}^{b} \psi_{a b}+\frac{2}{3} M M^{*}+\frac{1}{3} b^{a} b_{a}\right.
\end{aligned}
$$

$$
\begin{aligned}
& -i e_{a}^{m} \mathcal{D}_{m} b^{a}+\frac{1}{2} \bar{\psi} \bar{\psi} M-\frac{1}{2} \psi_{a} \sigma^{a} \bar{\psi}_{c} b^{c} \\
& \left.\left.+\frac{1}{8} \varepsilon^{a b c d}\left(\bar{\psi}_{a} \bar{\sigma}_{b} \psi_{c d}+\psi_{a} \sigma_{b} \bar{\psi}_{c d}\right)\right]\right\}
\end{aligned}
$$

Finally, the action of the chiral projector $\left(\overline{\mathcal{D}}_{\dot{\alpha}} \overline{\mathcal{D}}^{\dot{\alpha}}-8 R\right)$ on the field $\Phi^{\dagger}$ is given by [8]

$$
\begin{aligned}
& \left(\overline{\mathcal{D}}_{\dot{\alpha}} \overline{\mathcal{D}}^{\dot{\alpha}}-8 R\right) \Phi^{\dagger}= \\
& -4 F^{*}+\frac{4}{3} M A^{*}+\theta\left[-4 i \sqrt{2} \sigma^{c} \hat{D}_{c} \bar{\chi}-\frac{2}{3} \sqrt{2} \sigma^{a} b_{a} \bar{\chi}\right. \\
& \left.+\frac{4}{3} A^{*}\left(2 \sigma^{a b} \psi_{a b}-i \sigma^{a} \bar{\psi}_{a} M+i \psi_{a} b^{a}\right)\right] \\
& +\theta \theta\left\{-4 e_{a}^{m} \mathcal{D}_{m} \hat{D}^{a} A^{*}-\frac{8}{3} i b_{a} \hat{D}^{a} A^{*}\right. \\
& -\frac{2}{3} \sqrt{2} \bar{\psi}_{a b} \bar{\sigma}^{a b} \bar{\chi}+2 \sqrt{2} \bar{\psi}_{a} \hat{D}^{a} \bar{\chi}-\frac{8}{3} M^{*} F^{*} \\
& -\frac{2}{3} i \sqrt{2} \bar{\psi}_{a} \bar{\chi} b^{a}+\frac{1}{3} i \sqrt{2} \bar{\psi}_{a} \bar{\sigma}^{a} \sigma^{c} \bar{\chi} b_{c} \\
& +\frac{4}{3} A^{*}\left[-\frac{1}{2} \mathcal{R}+i \bar{\psi}^{a} \bar{\sigma}^{b} \psi_{a b}+\frac{2}{3} M M^{*}+\frac{1}{3} b^{a} b_{a}\right. \\
& -i e_{a}^{m} \mathcal{D}_{m} b^{a}+\frac{1}{2} \bar{\psi} \bar{\psi} M-\frac{1}{2} \psi_{a} \sigma^{a} \bar{\psi}_{c} b^{c} \\
& \left.\left.+\frac{1}{8} \varepsilon^{a b c d}\left(\bar{\psi}_{a} \bar{\sigma}_{b} \psi_{c d}+\psi_{a} \sigma_{b} \bar{\psi}_{c d}\right)\right]\right\}
\end{aligned}
$$

where

$$
\begin{aligned}
\hat{D}_{a} A^{*} & =e_{a}^{m} \partial_{m} A^{*}-\frac{1}{2} \sqrt{2} \bar{\psi}_{a \dot{\alpha}} \bar{\chi}^{\dot{\alpha}} \\
\hat{D}_{a} \bar{\chi}^{\dot{\alpha}} & =e_{a}^{m} \mathcal{D}_{m} \bar{\chi}^{\dot{\alpha}}-\frac{i}{2} \sqrt{2} \bar{\sigma}^{b \dot{\alpha} \delta} \psi_{a \delta} \hat{D}_{b} A^{*} \\
& -\frac{1}{2} \sqrt{2} \bar{\psi}_{a}^{\dot{\alpha}} F^{*} .
\end{aligned}
$$

\section{ACKNOWLEDGMENTS}

I sincerely thank Massimo Pietroni for many useful suggestions and discussions on Scalar-Tensor theories and their possible supersymmetric extensions. I would also like to thank Wilfried Buchmueller for interesting discussions on the topic and Massimo Pietroni and Gonzalo Palma for having read and commented on a draft of the paper. I finally acknowledges a Research Grant funded by the VIPAC Institute. 
[1] D. N. Spergel et al., [arXiv:astro-ph/0603449].

[2] W. de Boer, "Is dark matter supersymmetric?," Prepared for Cargese School of Particle Physics and Cosmology: the Interface, Cargese, Cosica, France, 4-16 Aug 2003.

[3] E. J. Copeland, M. Sami and S. Tsujikawa, [arXiv:hepth/0603057].

[4] T. Damour, "Gravitation, experiment and cosmology," [arXiv:gr-qc/9606079].

[5] P. Jordan, Schwerkaft und Weltall (Vieweg, Braunschweig, 1955); M. Fierz, Helv. Phys. Acta 29, 128 (1956); T. Damour and K. Nordtvedt, Phys. Rev. D48, 3436 (1993); T. Damour and A.M. Polyakov, Nucl. Phys. B423, 532 (1994); R. Catena, M. Pietroni and L. Scarabello,
[arXiv:astro-ph/0604492]

[6] N. Bartolo and M. Pietroni, Phys. Rev. D 61 (2000) 023518 [arXiv:hep-ph/9908521]; G. Esposito-Farese and D. Polarski, Phys. Rev. D 63, 063504 (2001) [arXiv:grqc/0009034]; R. Catena, N. Fornengo, A. Masiero, M. Pietroni and F. Rosati, Phys. Rev. D 70, 063519 (2004) [arXiv:astro-ph/0403614].

[7] C. Brans and R. H. Dicke, Phys. Rev. 124 (1961) 925.

[8] Julius Wess and Jonathan Bagger, Supersymmetry and Supergravity, Princeton University Press.

[9] B. Bertotti, L. Iess and P. Tortora, Nature 425 (2003) 374. 